\title{
Stability and Hopf Bifurcation Analysis in a Delayed Gause-type Predator-prey Models
}

\author{
Shuang Guo, Xing Qiao*, Dongxia Zhao, Lianguang Jia and Xiuyan Jiang \\ School of Mathematical Sciences, Daqing Normal University, Daqing 163712, China \\ ${ }^{*}$ Corresponding author
}

\begin{abstract}
A class of delayed ratio-dependent Gause-type predator-prey model is considered. Firstly, the eigenvalue problem is studyed for the linearized system at the coexisting equilibrium and a group of sufficient conditions for the existence of Hopf bifurcation are obtained. Secondly, the direction of the Hopf bifurcation and the stability of the bifurcated periodic solutions are determined by applying the normal form method and the center manifold theorem. Finally, some numerical simulations are carried out to illustrate the obtained results.
\end{abstract}

Keywords—gause-type model; stability; hopf bifurcation

\section{INTRODUCTION}

We know that the population quantity may happen the perio-dic fluctuation in nature. The influence of time delay can be received from the view of mathematics, See [1-7]. In 1998, Beretta and Kuang [5] lead a single discrete time delay to predator equation of the following model:

$$
\left\{\begin{array}{l}
x^{\prime}=a x\left(1-\frac{x}{K}\right)-\frac{c x y}{m y+x} \\
y^{\prime}=y\left(-d+\frac{f x(t-\tau)}{m y(t-\tau)+x(t-\tau)}\right)
\end{array}\right.
$$

where $x$ and $y$ are the population densities of prey and predator at time $t$, respectively. $a, K, c, m, f, d$ are positive constants that stand for prey intrinsic growth rate, carrying capacity, capturing rate, half capturing saturation constant, conversion rate, predator death rate, respectively. For this model, the authors studied the stability of periodic solutions and the existence of Hopf bifurcation employing $\tau$ as the bifurcation parameter. Ginoux [8] highlighted this model has several Hopf bifurcations and a period doubling cascade generating a snail shell-shaped chaotic attractor. They compared the quantity and property of the equilibrium, bifurcation structure and shape of attractors among this model, and also gave the bifurcation analysis for each model. In order to prove the global asymptotic stability of the positive equilibrium, some sufficient conditions are given by constructing appropriate Lyapunov functions.

The purpose of current work is to analysis the effect of delay on the dynamics for the following delay ratio-dependent food chain model:

$$
\left\{\begin{array}{l}
x^{\prime}=\operatorname{ax}\left(1-\frac{x}{K}\right)-\frac{c x y}{m y+x} \\
y^{\prime}=y\left(-d+\frac{f x}{m y+x}-r z\right) \\
z^{\prime}=z(-s+e y(t-\tau))
\end{array}\right.
$$

where $Z$ is the population densities of top predator at time $t$. $r, e, s$ are positive constants that stand for capturing rate, half capturing saturation constant, conversion rate, top-predator death rate, respectively. In [9-12], the authors reveal that Hopf bifurcation can occur as the delay crosses some critical values which leads to the existence of periodic solution that may conform to certain phenomena in ecosystem system.

We still let $\tau$ as the bifurcation parameter in this paper and consider the delay Gause-type predator-prey model with ratio-dependent functional response. The rest of the paper is organized as follows: Firstly we investigate the stability of coexisting equilibrium and the existence of the Hopf bifurcation of the equation (2) by analyzing the characteristic equation of the associated linearized system. Then we carry out some numerical simulations to illustrate the results obtained and forecast the change of population quantity.

\section{StABILITY AND Hopf BIFURCATION OF COEXISTING EQUILIBRIUM}

For the sake of convenience, we non-dimensionalizes (2), then (2) takes the form:

$$
\left\{\begin{array}{l}
x^{\prime}=x(1-x)-\frac{p x y}{x+y} \\
y^{\prime}=y\left(-l+\frac{q x}{x+y}-z\right) \\
z^{\prime}=z(-s+u y(t-\tau))
\end{array}\right.
$$

which satisfies $x_{0}(\theta)=\phi_{1}(\theta) \geq 0, y_{0}(\theta)=\phi_{2}(\theta) \geq 0$, 
$z_{0}(\theta)=\phi_{3}(\theta) \geq 0, \theta \in[-\tau, 0], x(0)>0, y(0)>0$, $z(0)>0$ and $\phi(\theta)=\left(\phi_{1}, \phi_{2}, \phi_{3}\right) \in C\left([-1,0], R^{3}\right),\|\phi\|=$ $\max \{\phi(\theta): \theta \in[-\tau, 0]\},|\phi|$ is any norm in $R^{3}$.And

$$
p=\frac{c}{m a}, q=\frac{f}{a}, l=\frac{d}{a}, u=\frac{e k}{m} .
$$

Obviously, the delay can't change the number of equilibria and non-dimensionalizes can't change the properties of system.

In the following, we always assume (3) has a positive Equilib-rium exists and denote it by $\bar{E}=\left(x^{*}, y^{*}, z^{*}\right)$ with

$$
\begin{gathered}
x^{*}=\frac{\left(1-\frac{s}{u}\right)+\sqrt{\left(1-\frac{s}{u}\right)^{2}+\frac{4 s(p-1)}{u}}}{2}, \\
y^{*}=\frac{s}{u}, z^{*}=\frac{q x^{*}}{x^{*}+y^{*}}-l
\end{gathered}
$$

We consider the linearized system of (3) at $\bar{E}$. The characteristic equation at $\bar{E}$ is given by

$$
D(\lambda, \tau)=\lambda^{3}+a_{2} \lambda^{2}+a_{1} \lambda+\left(b_{1} \lambda+b_{0}\right) e^{-\lambda \tau}=0,
$$

where

$$
a_{2}=-m_{11}-m_{22}, a_{1}=m_{11} m_{22}-m_{12} m_{21}, b_{1}=-m_{23} n_{32},
$$

and $b_{0}=m_{11} m_{23} n_{32} \cdot m_{11}=1-2 x^{*}-\frac{p y^{*^{2}}}{\left(x^{*}+y^{*}\right)^{2}}$,

$m_{12}=-\frac{p x^{*^{2}}}{\left(x^{*}+y^{*}\right)^{2}}<0, m_{23}=-y^{*}<0, n_{32}=u z^{*}>0$.

$$
m_{21}=\frac{q y^{*^{2}}}{\left(x^{*}+y^{*}\right)^{2}}>0, m_{22}=-\frac{q x^{*} y^{*}}{\left(x^{*}+y^{*}\right)^{2}}<0,
$$

If $m_{11}<0$, then all the eigenvalues of (4) have negative real parts when $\tau=0$ by the Routh-Hurwitz criterion. Now we substitute $\lambda=i \omega(\omega>0)$ into (4),
(1) when $\omega=0, D(0, \tau)=b_{0}=m_{11} m_{23} n_{32} \neq 0$;

(2) when $\omega \neq 0$,

$$
D(i \omega, \tau)=(i \omega)^{3}+a_{2}(i \omega)^{2}+a_{1} i \omega+\left[b_{1}(i \omega)+b_{0}\right] e^{-i \omega \tau}=0 .
$$

Separating the real and imaginary parts gives

$$
\begin{gathered}
-a_{2} \omega^{2}+b_{0} \cos \omega \tau+b_{1} \sin \omega \tau=0, \\
-\omega^{3}+a_{1} \omega+b_{1} \omega \cos \omega \tau-b_{0} \sin \omega \tau=0 .
\end{gathered}
$$

We get

$$
\omega^{6}+\left(a_{2}^{2}-2 a_{1}\right) \omega^{4}+\left(a_{1}^{2}-b_{1}^{2}\right) \omega^{2}-b_{0}^{2}=0 .
$$

Denote $h(l)=l^{3}+P_{1} l^{2}+P_{2} l+P_{3}=0$. (7) has one positive root at least because $h(0)=P_{3}<0$. Without loss of general-ity, we assume that it has three positive roots, denoted by $l_{1}, l_{2}, l_{3}$, respectively. Then (3) has three positive roots $\omega_{i}=\sqrt{l_{i}}(i=1,2,3)$. We can calculate

$$
\tau_{i}^{(k)}=\frac{1}{\omega_{i}}\left\{\arcsin \frac{\left(a_{2} b_{1}-b_{0}\right) \omega_{i}^{3}+a_{1} b_{0} \omega_{i}}{b_{1} \omega_{i}^{2}+b_{0}^{2}}+2 k \pi\right\},
$$

$k=0,1,2 \cdots \cdots$ Define

$$
\tau_{0}=\min \left\{\tau_{i}^{(0)}\right\}, \quad \omega_{0}=\omega_{i},
$$

Denoting $\lambda(\tau)=\alpha(\tau)+i \beta(\tau)$ be the root of (3) satisfying $\alpha\left(\tau_{0}\right)=0, \beta\left(\tau_{0}\right)=\omega_{0}$, we have the following lemma.

Lemma If $h\left(\omega_{0}^{2}\right) \neq 0$, we have $\frac{d \operatorname{Re} \lambda(\tau)}{d \tau}>0$.

Proof :Assume

$$
h\left(\omega_{0}^{2}\right)=\omega_{0}^{6}+\left(a_{2}^{2}-2 a_{1}\right) \omega_{0}^{4}+\left(a_{1}^{2}-b_{1}^{2}\right) \omega_{0}^{2}-b_{0}^{2},
$$

So $h^{\prime}\left(\omega_{0}^{2}\right)=3 \omega_{0}^{4}+2\left(a_{2}^{2}-2 a_{1}\right) \omega_{0}^{2}+\left(a_{1}^{2}-b_{1}^{2}\right)$, because 


$$
\begin{aligned}
& \left.\operatorname{sign}\left[\frac{d \operatorname{Re} \lambda(\tau)}{d \tau}\right]\right|_{\tau=\tau_{0}}=\left.\operatorname{sign}\left[\frac{d \operatorname{Re} \lambda(\tau)}{d \tau}\right]^{-1}\right|_{\tau=\tau_{0}}, \\
& {\left.\left[\frac{d \operatorname{Re} \lambda(\tau)}{d \tau}\right]^{-1}\right|_{\tau=\tau_{0}}+\left.\operatorname{Re}\left[\frac{b_{1}}{b_{1} \lambda^{2}+b_{0} \lambda}\right]\right|_{\tau=\tau_{0}}} \\
& =\left.\operatorname{Re}\left[\frac{\left(3 \lambda^{2}+2 a_{2} \lambda+a_{1}\right) e^{\lambda \tau}}{b_{1} \lambda^{2}+b_{0} \lambda}\right]\right|_{\tau=\tau_{0}}+ \\
& =\frac{3 \omega_{0}^{4}+2\left(a_{2}^{2}-2 a_{1}\right) \omega_{0}^{2}+\left(a_{1}^{2}-b_{1}^{2}\right)}{b_{1} \omega_{0}^{2}+b_{0} \omega_{0}^{2}} \\
& =\frac{h^{\prime}\left(\omega_{0}^{2}\right)}{b_{1}^{2} \omega_{0}^{2}+b_{0}^{2} \omega_{0}^{2}} .
\end{aligned}
$$

We have $h\left(\omega_{0}^{2}\right) \neq 0$, then $\left.\frac{d \operatorname{Re} \lambda(\tau)}{d \tau}\right|_{\tau=\tau_{0}} \neq 0$.There must be $\left.\frac{d \operatorname{Re} \lambda(\tau)}{d \tau}\right|_{\tau=\tau_{0}}>0$. This is because (3) has the positive real part roots as $\tau<\tau_{0} \quad$ if $\left.\frac{d \operatorname{Re} \lambda(\tau)}{d \tau}\right|_{\tau=\tau_{0}}<0$. This contradicts to the fact when $\tau \in\left[0, \tau_{0}\right)$ and $\bar{E}$ is asymptotically stable [13].

By Lemma we have the following theorem.

Theorem Suppose that $m_{11}<0$. If Lemma holds, then the equilibrium $\bar{E}=\left(x^{*}, y^{*}, z^{*}\right)$ of the delay model (2) is asym-ptotically stable when $\tau<\tau_{0}$, and unstable when $\tau>\tau_{0}$, where $\tau_{0}$ is defined by (8). In addition, if $h\left(\omega_{0}^{2}\right) \neq 0$, then Hopf bifurcation occurs when $\tau=\tau_{0}$.

\section{NUMERICAL SimUlations AND Discussions}

In this part, we perform some numerical simulations. The results not only support our previous parts, but also predict the existence of global Hopf bifurcations periodic solutions. However, to prove this observation theoretically is of great challenge.

We choose the parameters as follows:

$$
\begin{aligned}
& \quad p=0.862, q=0.199, l=0.1182, r=0.12, \\
& s=0.4145, u=0.629, \text { thus through (8), we have } \\
& \tau_{0}=2.164 \text { and } c_{1}(0)=-0.1761+i 0.5602 \text {. Utilizing }
\end{aligned}
$$
Theorem, we know the equilibrium of the delay model (2) is asymptotically stable

When $\tau<\tau_{0}$ (see Figure. 1).Hopf bifurcation occurs when $\tau=\tau_{0}$, and the bifurcating periodic solution is orbitally asymptotically for $\tau>\tau_{0}$ (see Figure. 2).

In addition, the periodic solution of system (2) still exist when $\tau$ is large and its amplitude is larger compared with thesolution. How to explain the phenomenon theoretically needs further researches.
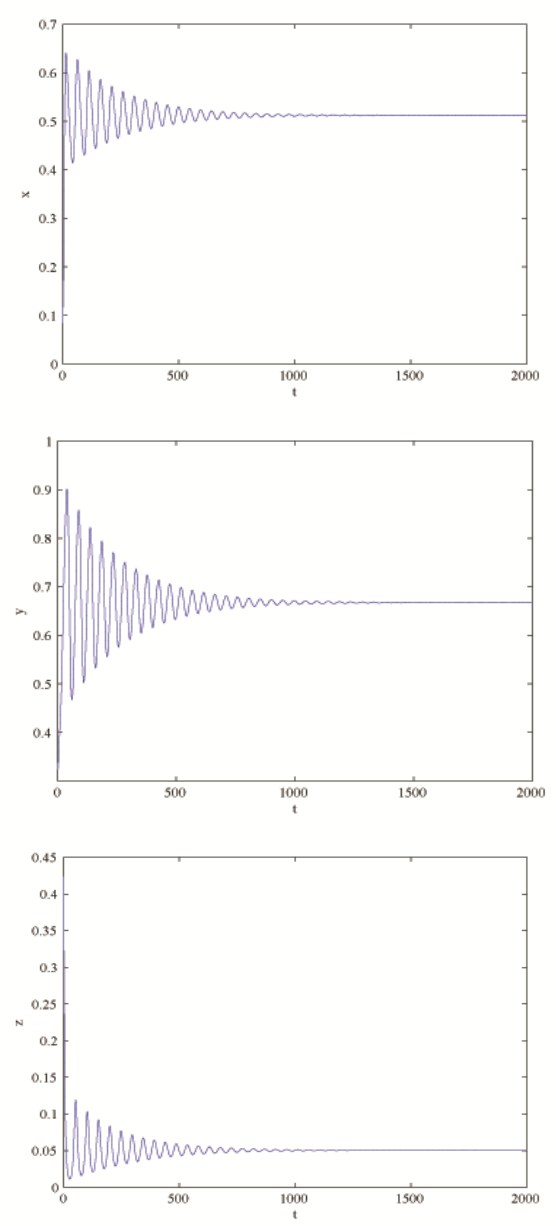


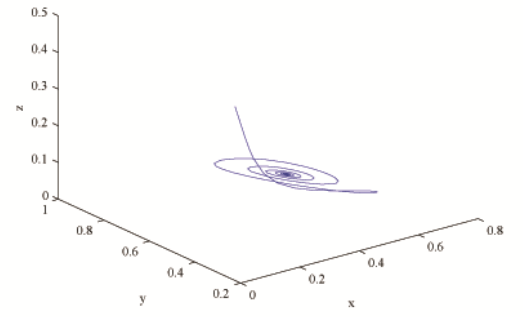

FIGURE I. $\bar{E}(0.5134,0.6755,0.0637)$ IS ASYMPTOTICALLY STABLE WHEN $\tau=2.065<\tau_{0}=2.164$.
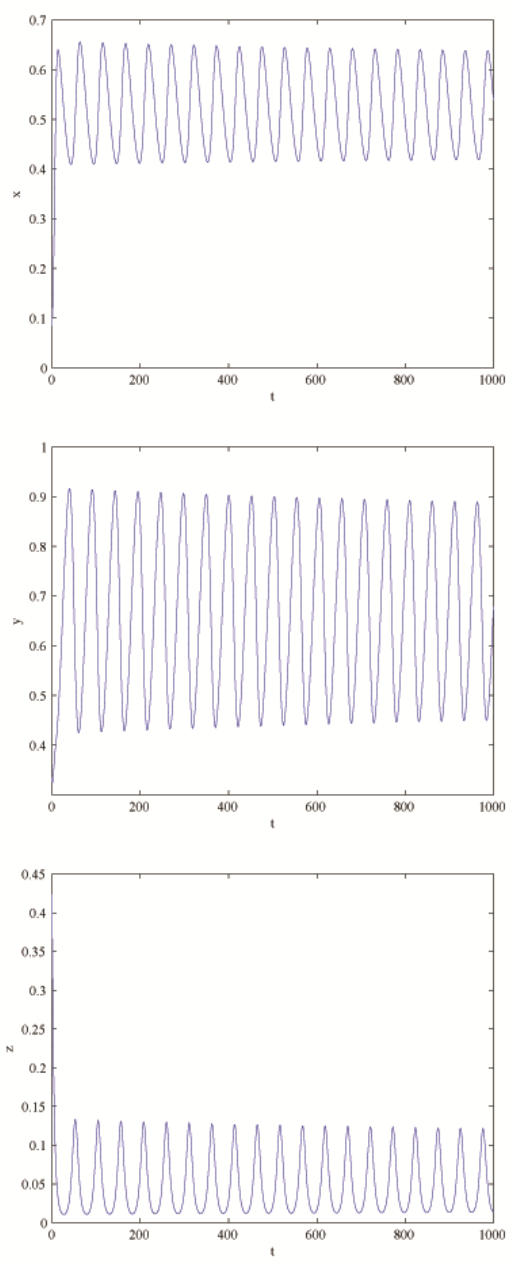

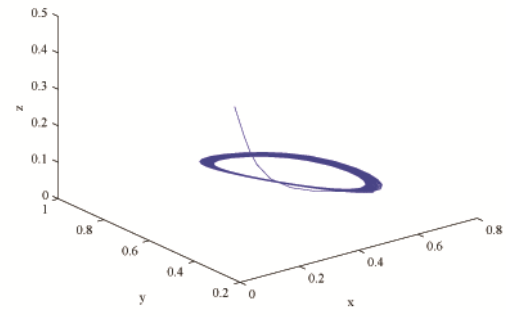

FIGURE II. A PERIODIC ORBIT OF SYSTEM NEAR $\bar{E}(0.5134,0.6755$, 0.0637) WHEN $\tau=2.567>\tau_{0}=2.164$

\section{CONCLUSION}

In this paper, we analyze the dynamics of the equilibria coexistence for a class of three-dimensional Gause-type predator-prey model. We obtain the stability of this equilibrium and also claim that the introduced delay changes its stability while a Hopf bifurcation occurs. The bifurcation periodic solutions also existence for sufficiently large delay.

\section{ACKNOWLEDGMENT}

The work is supported by the PhD Start-up Daqing Normal University (No.14ZR09) and the Natural Science Foundation of Daqing Normal University (No.14ZR07).

\section{REFERENCES}

[1] A. Berryman, “The origins and evolution of predator-prey”, Ecology, vol. 73, 1992, pp.1530-1535.

[2] H. I. Freedman, Deterministic Mathematical Models in Population Ecology, New York: Marcel Dekker Incorporated, Chap. 2, 1980.

[3] R. Arditi and L. R. Ginzburg, "Coupling in predator-prey dynamics: ratio dependence”, J. theor. Biol., vol. 139, 1989, pp.311-326, 1989.

[4] Y. Kuang and E. Beretta, "Global qualitative analysis of a ratiodependent predator-prey system”, J. Math. Biol.,vol. 36, 1998, pp. 389406.

[5] E. Beretta and Y. Kuang, "Global analysis in some delayed ratiodependent predator-prey system”, Nonlinear Anal-theor, vol. 323,1998, pp.381-408.

[6] R. Arditi and A. A. Berryman, "The biological control paradox", Ttends Ecol., vol. 6, 1991,pp. 32.

[7] T. S, Yi and X. F. Zou, " Global dynamics of a delay differential equation with spatial non-locality in an unbounded domain”, J. Differ Equations, vol. 251, 2011, pp. 2598-2600.

[8] J. M. Ginoux, B. Rossetto and J. L. Jamet, "Chaos in a three-dimensional Volterra-Gause model of predator-prey type”, Internat. J. Bifur. Chaos, vol. 15, 2005, pp. 1689-1708.

[9] M . Freeze, Y. Chang and W. Feng, "Analysis of Dynamics in a Complex Food Chain with Ratio-Dependent Functional Response”, J. Appl. Anal. Comp., vol. 4, 2014, pp. 69-87.

[10] S. G. Ruan and J. J. Wei, "On the zeros of a third degree exponential polynomial with applications to a delayed model for the control of testosterone secretion”, Math. Med. Biol., vol. 18,2001, pp.41-52.

[11] W. H. Jiang and J. J. Wei, "Bifurcation analysis in a limit cycle oscillator with delay feedback”, Chaos, Solitons Fract, vol. 23, 2005, pp.817-831.

[12] H. B. Wang and W. H. Jiang, "Hopf-pitchfork bifurcation in van der Pol's oscillator with nonlinear delayed feedback”. J. Math. Anal. Appl., vol. 368, 2010, pp. 9-18. 
[13] B. Hassard, N. Kazarinoff, and Y. H. Wan, Theory of Application of Hopf Bifurcation. London: Cambridge University, Chap. 3, 1981. 